\title{
ENFERMEDAD DE TAKAYASHU
}

\author{
Prof. ADALBERTO STEPGER Y Dr, LAUTARO VARGAS \\ Catedra de Pediatría del Prof. Adalberto Steeger \\ Hospital "San Juan de Dios". Santiago.
}

La enfermedad de Takayashu pertenece al síndrome del anco aórtico descrito por Ross y Me Kusick ${ }^{20}$ en 1953 que engloba a todas aquellas afecciones en las que hay obliteración total o parcial de las arterias del cayado aórtico y que se expresan por una sintomatología común. derivada de la isquemia de la mitad superior del cuerpo, a saber: 1) hipotensión y ausencia o disminución del pulso radial y carotideo (por compromiso de las subclavias) y 2) síntomas cerebrales y especialmente oculares (por compromiso de las carótidas). Otro becho importante, consecuencia del anterior, es el desarrollo de una circulación colateral que lieva sangre de la aonta descendente a los territorios isquemiados.

Una de las múltiples causas de este síndrome es la enfermedad de Takayashu, afección estudiada especialmente en $\mathrm{Ja}$ pón ${ }^{17}$, donde se la describió por primera vez. Desde 1950 se están comunicando ca-

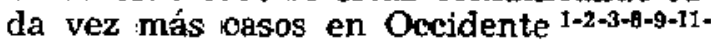
12-13. En la literatura pediátrica, las referencias a esta enfermedad son escasas ${ }^{5}$. El paciente que presentamas es el primero de esta indiole en Chile, y sólo hay descritos dos casos en adultos ${ }^{13}$, por lo que creemos oportuno hacer ana breve revisión de los conceptos fundamentales de la enfermediad.

Esta enfermedad ha recibido numerosos nombres: enfermedad sin pulso, sindrome de Martorell-Fabré, coartación de la aorta invertida ${ }^{16}$, trombosis de las ramas del cayado aórtico ${ }^{3.17}$, etc.

Se podría definir como una enfermedad de curso crónico, más frecuente en mujeres jóvenes, y en la que existe una panarteritis con trombosis, de etiologia desconocida, de los vasos del cayado aórtico, a consecuencia de la cual se produce un défjcit de la irrigación de la mitad superior del cueripo.

El mayor número de casos se encuientra entre los 15 y los 45 años (término medio, 30 años). Se han descrito aligunos casos en individuos de menor edad. Colón Rivera " comunica el caso de una niña de 5 años. Más o menos el $80 \%$ de los casos corresponde a mujeres.

\section{ANATOMÍA PATOLógica}

La anatomía patológica ${ }^{1.3}$ de esta enfermedad está caracterizada por una panarteritis obliterante de las ramas del cayado aórtico y por el desarrollo de un sistema colaterali a través de las arterias in tercostales, mamarias internas y vertebrales. La extensión de la arteritis es variable; puede afectar al tronco braquicefálico, carótida izquierda o subclavia izquierda, separadamente o en forma progresiva. Las lesiones se inician en el nacimiento de los vasos o a algunos centímetros de su origen. Por las carótidas se propaga hasta el seno carotídeo, rara vez más allá, llegando hasta la base del cráneo. Generalmente las arterias humerales y cerebrales están indemnes. Hacia abajo, las lesiones no van más allá de la primera porción de la aorta torácica. En un caso de Ash Upmark ${ }^{2}$ había lesiones de las arterias mesentérica y celíaca. En algunos casos se ha encontrado hipertrofia del ventrículo izquierdo $\mathrm{y}$ en otro riñones hipoplásicos. La panarteritis lleva tarde o temprano a la obliteración de los vasos por trombosis, aunque a veces se produce recanalización de estos. Histológicamente hay proliferación de la íntima y de la media, con infiltración linfo-plasmocitaria, especialmente de los vaso vasorum. A veces se eneuentran células gìgantes.

\section{E'TIOLOGÍA}

La etiología de la enfermedad se desconoce. Algunos autores invocan el origen infeccioso ${ }^{3}$, a favor del cual estaría la presencia de fiebre, velocidad de sedimentación elevada y presencia de antiestreptolisina en algunos casos. Sin embango, no se ha encontrado nunca ningún 
germen en las lesiones arteriales ni en hemocultivos; igualmente el tratamiento con antibióticos ha fracasado.

Otros creen en una etiologia aléngica, debido a la acción favorable de los corticoides y a la similitud que tiene esta enfermedad con otras angeítis aléngicas (angeítis por hipersensibilidad, angeítis alérgica granulomatosa, poliarteritis nodosa).

Por úlltimo, se podiría unir estas 2 hipótesis en una ${ }^{\text {B.13 }}$; pensando en que la infección podiría ser el factor desencadenante que determine una reacción inmunoslérgica, algo así como en la enfermedad reuimática. Otros autores asimilan esta enfermedad a las enfermedades del mesénquima; sin embargo, en estas, las lesiones son generalizadas.

\section{CUADRO CLÍNICO}

Respecto al cuadro clínico, siguiendo a Ash Upmark, dividiremos la sintomatología en: 10) sintomas derivados de la isquemia de la mitad superior del cuerpo; $2^{9}$ ) hipersensibilidad del seno carotídeo; $3^{\circ}$ ) desanrollo de cirexiación colateral; $4^{\circ}$ ) síntomas cardíacos y $5^{\circ}$ ) síntamas generales.

19) Síntomas derivados de la isquemia de la mitad superior del cuerpo.

a) Por compromiso de las subclavias. Se produce un signo fundamental cual es la ausencia o disminución del pulso de las arterias radiales, humerales, axilares y carotídeas. La tensión arterial ibaja consecuentemente; la diferencial está neducida, de modo que la presión media está más o menos conservada, lo que explicaría la falta de síntomas tróficos. La presión sistólica oscila generalmente en cifras de 80 a $75 \mathrm{~mm}$. Hg. A veces no se puede tomar la presión por el método auscultatorio; en estos casos se debe usar el método de Gaertner que consiste en insuflar el manguito teniendo el brazo levantado por encima de la cabeza, luego se baja el brazo y se va reduciendo la presión lentamente, hasta que aparece una coloración rosada en la mano y antebrazo que indica la presión arterial media.

En los miembros inferiores se palpan los pulsos pedios normales y a veces, la presión arterial es alta, lo que ha hecho a algumos autores Ilamar a esta enfermedad coartación de la aorta invertida.
En algunos enfermos hay hipotensión arterial de un brazo e hipertensión en el otro.

Los enfermos relatan parestesias, debilidad y cansancio rápido de las extremidadies superiores.

b) Por compromiso de las carótidas. Sintomas oculares 8 -18. Son muy importantes ya que la arteria central de la retina es una arteria terminal. Los sintomas subjetivos son variados: amaurosis transitoria uni o bilateral, visión borrosa, disminución de la agudeza visual, fotopsias.

Objetivamente, se encuentran cataratas (casos extremos) y en el fondo de ojos puede existir atrofia retinal y coroidea, anastomosis peripapilares arterio venosas, flujo lento de los vasos de la retina. Estrechamiento del campo visual. La presión de la arteria central de la retina está muy disminuída.

Síntomas encefálicos. Estados vertiginosos, cefaleas, accidentes encefálicos: hemiparesia, hemiplegia, afasia, convulsiones.

Sintomas auditivos. Tinitus, sordera.

Síntomas faciales. Cansancio de los músculos masticadores. Atrofia facial, atrofia de los procesos alveolares con pérdida de los dientes, penforación del tabique nasal, ulceración del paladar (esto se presenta en casos muy avanzados y muy rara vez).

$2^{\circ)}$ Síntomas derivados de la hipersensibilidad del seno carotídeo. Están presentes sólo en algunos enfermos y se traducen por síncopes con los cambios de posición o al comprimir el seno carotídeo, se deberian a la acción del tejido cicatricial que se produce alrededor del mismo.

$3^{\circ)}$ Síntomas derivados del desarrollo de la circulación colateral. Se manifiestan por arterias superficiales palpables, erosiones costales y auscultación de soplos que generalmente son audibles en el ángulo esterno-clavicular, y en su gran mayoría sistólicos.

La cinculación colateral se realiza a través de las mamarias internas, intercostales, vertebrales; en igual forma que en la coartación aórtica, pero en una dirección inversa: la sangre va deside la aorta descendente a las ramas del cayado aórtico.

49) Síntomas cardiovasculares. En algunos casos existe taquicardia, hiperten- 
sion arterial de las extremidades inferiores y a veces del brazo no comprometido. No se conoce una explicación valedera para esta hipertensión; según algunos podría deberse a isquemia diencefálica.

50) Síntomas generales. A veces hay fiebre, velocidad de sedimentación elevada.

\section{EXÁMENES DE LABORATORIO}

1) La radiografía de tórax puede demostrar erosiones costales e hipertrofia del ventrículo izquierdo.

2) La angiocardiografía demuestra la falita de impregnación por el medio de contraste de los grandes troncos del cayado y permite ver la importancia de la red colateral.

3) El electroencefalograma puede dar alteraciones inespecíficas.

4) En algunos enfermos pueden existir alteraciones de la relación albúminaglobulina.

5) La biopsia arterial no sirve, ya que los vasos accesibles (subclavia, humeral) generalmente no están alterados.

\section{DIAGNÓSTICo}

Se debe plantear el diagnóstico diferencial "n con todas las demás causas del síndrome aórtico, que se acompañan de ausencia de pulso radial o carotídeo. Una vez descartadas todas estas causas, se puede plantear el diagnóstico de enfermedad de Takayashu, ya que carecemos de un test específico y de la posibilidad de biopsia que permita certificar el diagnóstico.

El diagnóstico debe ser por exclusión de las siguientes enfermedades fácilmente descartables y que sólo enumeraremos: 1) aortitis sifilítica con o sin aneurisma; 2) arterioesclerosis; 3) aneurisma traumático; 4) causa congénita (generalmente coartación de la aorta con estenosis de la subclavia asociada); 5) aneurisona disecante crónico; 6) embolía arterial; 7) trombofilia; 8) tumor del mediastíno; 9) costilla cervical; 10) síndrome diel escaleno; 11) poliarteritis nodosa; 12) Enfermedad de Leo Bürger.

\section{EVOLUCIón}

Es una enfermedad de curso crónico, progresivo. Una vez hecho el diagnóstico Ia sobrevida varía entre 1 a 16 años. La mayoría de los casos evoluciona entre 3 y $\tilde{\jmath}$ años. La muerte se produce generalmente por accidentes vasculares encefálicos.

\section{TRATAMIIENTO}

Médico. A base de anticoagulantes y corticoides. En algunos casos se ha obtenido éxito, en otros el resultado es discutible y contradictorio. El tratamiento debe ser de langa duración.

Quirúrgico. Sus posibilidades son discutibles, ya que es una enfermedad progresiva, aunque según De Bakey ${ }^{7.19}$ se debe intentar, ya que en muchos casos las trombosis son localizadas como en el síndrome de Lériche. Habrían 3 posibilidades quirúrgicas: 1) Tromboendarterectomía; 2 ) Resección del segmento trombosado y reemplazo por injerto, y 3 ) colocación de un injerto que sirva de derivación (bypass) saltándose en esta forma el sitio de la obstrucción. Se han comunicado algunos casos operados con éxito, y controlados durante 1 o 2 años.

\section{PRESENTACIÓN DEL CASO}

N. B. P. (Obs. 31511). 11 años 6 meses.

Se hospitaliza por primera vez (2-V-573-IX-57) por una hemorragia digestiva masiva que no cedió a múltiples transfusiones por lo cual se intervino en 2 ocasiones, encontrándose en la segunda in tervención un vaso roto del fondo gástrico que fué suturado sin inconvenientes. Ulteriormente no ha vuelto a sangrar. Además, un examen radiolóngico de tubo digestivo de Marzo de 1960 fué normal. Una vez que se solucionó el problema de la hemorragia se evidenció una hipertensión arterial que fluctuó entre 170-140/ 100-90. Se descartaron las siguientes etiologías de la hipertensión: 1) nefropatía médica (por normalidad de exámenes de orina, recuento de Addis, prueba de Volhard); 2) Feocromocitoma (prueba de la histamina negativa); 3 ) Coartación aórtica (por la existencia de pulsos pedios y femorales presentes). Se dió de alta con diagnóstico de Enfermedad Hipertensiva Esencial.

Su segunda hospitalización fué del 25IX-58 al 9-II-58. Desde el alta anterior solo habia presentado cefaleas frontales ocasionales. Se hospitaliza para completar el estudio de la hipertensión, la cual 
seguía con cifras de 170-120/110-80 en el brazo derecho. En el brazo izquierdo no se encontró presión por el método auscultatorio. Se descartó riñón quirúrgico por examen de pielografía descendente, que fué nonmal; los exámenes de orina y de función renal seguian normales.

En Diciembre de 1958 se realizó una angiocardiografía (Hospital "Luis Calvo Mackenna") que demostró falta de contraste de la arteria subclavia izquierda.

La tercera hospitalización (19-I-60-5-II60) se debió a que la enferma se quejaba de una intensificación de su cefalea, la cual se había hecho más frecuente y se habían agregado mareas, visión borrosa después de leer, lipotimias en 2 ocasiones y parestesias de las manos. El examen fisico mostraba lo siguiente: relativo buen estado general, rendimiento intelectual normal, talla 1,58, peso $42 \mathrm{kggs}$. El examen cardíaco era normal. Había un suave soplo sistólico carotídeo izquierdo. Los hallazgos más importantes estaban en los pulsos y las presiones:

\begin{tabular}{|c|c|c|}
\hline Pulsas: & Derectio: & Izquterdo: \\
\hline $\begin{array}{l}\text { Fadlal } \\
\text { Fumerai } \\
\text { Carotideo } \\
\text { Fenorales y pedios }\end{array}$ & $\begin{array}{l}\text { muy dismin. } \\
\text { disminuido } \\
\text { normel } \\
\text { normal }\end{array}$ & $\begin{array}{l}\text { gusente } \\
\text { dlarninuido } \\
\text { algo dismin. } \\
\text { normal. }\end{array}$ \\
\hline \multicolumn{3}{|l|}{ Presion arterlal: } \\
\hline Humeral & $95 / 60$ & $\begin{array}{l}60 \text { mm. (prealón } \\
\text { medis, motodo } \\
\text { Caertner) }\end{array}$ \\
\hline Muslos & $I 30 / 60$ & $120 / 00$ \\
\hline \multicolumn{3}{|l|}{ oxcllometria: } \\
\hline $\begin{array}{l}\text { Mufeca } \\
\text { Brazo }\end{array}$ & $\begin{array}{l}0,5 \\
1,5\end{array}$ & \\
\hline
\end{tabular}

Con estos hallazgos y ante la evidencia de la evolución de las cifras tensionales que de 170-120/110-80 bajan a 95/60 se piensa en una enfenmedad evolutiva que afecta las ramas del cayado aórtico, lo que nos explicaría la falta de pulsos y la hipotensión arterial del brazo izquierdo; esta suposición estaría comprobada por la angiocardíografía que demostró compromiso de la subelavia izquierda, ya que era fácilmente descartable la arterioesclerosis y la sífilis, las dos principales causas del síndrome del arco aórtico; pensamos en la prosibilidad de una enfermedad de Takayashu, más aún tratándose de una mujer joven. Se pidió el examen oftalmológico, que reveló una hipotensión acentuada de la arteria central de la retina (mé- todo de Baillant) ojo derecho: $20 / 40$, ojo izquierdo: 20/35 (nommal 35/70). En el campo visual habia un marcado estrechamienta irregular del ojo izquierdo y en el fondo de ojo se encontraban pequeños focos blanquecinos en ambos ojos, que corresponderían a procesos de atrofia coroidea. El examen del $8^{\circ}$ par rama vestibular dio hipoexcitabilidad a izquierda. Audiograma normal. Otros exámenes fueron normales: Kahn, Colesterol, uremia, hemograma.

En esta enferma hay elementos suficientes para hacer el diagnóstico de Síndrome del Anco Aórtico: 1) Ausencia y disminución de los pulsos e hipotensión arterial de los miembros superiores (ambos hechos más acentuados a izquierda), 2) síntomas que podrian atribuirse a la esfera encefálica (mareos, cefaleas, lipotimias) y oculares (visión borrosa) más los hallazgos que demuestran compromiso dé la irrigación ocular: hipotensión de la arteria central de la retina, alteración del campo visual, alteraciones en el fondo del ojo y 3) una angiocardiografía del año 1958 que demuestra una estenosis de la arteria subclavia izquierda. El diagnóstico de enfermedad de Takayashu lo fundamos en que es una nifia joven en la que son fácilmente descartables las otras causas del síndrome del arco aórtico (sífilis, arterioesclerosis, traumatismo, causa congénita, etc.).

De acuendo con este diagnóstico se instauró tratamiento con Meticortén y anticoagulantes (dicumarol); se ha usado Meticortén en dosis decrecientes hasta llegar a una dosis de mantenimiento de $5 \mathrm{mg}$.; pensamos mantener este tratamiento un tiempo largo según respuesta de la enferma. Actualmente está asintomática y los signos clínicos de abstrucción arterial no han progresado.

\section{RESUMGN}

Se presenta un caso de Enfermedad de Takayashu y se hace una revisión de los conceptos fundamentales de la enfermedad, del diagnóstico diferencial y de su tratamiento.

\section{SUMMAFY}

\section{TAKAYASHU'S DISEASE}

A description is made on a case of $\mathrm{Ta}$ kayashu's disease. On this subject-matter 
a reviewing is done about the funda. mental concepts of this sickness, differential diagnosis and treatment.

\section{BIBLIOGRAFÍA}

1, $\rightarrow$ ASK UPMANK, In, - On the Pulaeless Drsease outside of Japan. Acte Med. scandinav. 149:161, 1954.

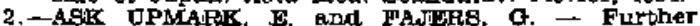
Obsemvations on Takayastiv's Disease. Acte Med. Scandinevian. 155, 275, 1956.

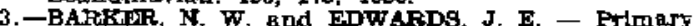
Arterlt16 of the Aorth Arah. Girculation. 11:480. 1855.

4.-BORDPT, F. - A Propos de l'enlgme des temmes tens pouls. Arch. Mal. Coour. 48:1105, 1955.

5. Cotlón Fivitra, E. - Pliseless Disease, prellminary fleport. Bol, Asoc. Mect. Puerto Rico. 47: $76, \mathrm{I} 955$.

6. COSME MAFUTAMA, B!OFIT sease. C1rculation. 20:267, 1959. DE HAXBY, M. et al. - Segmental thromboobliterative Disease of Branches of Aortle Arch. J. A. M. A 165:0;8, 1058.

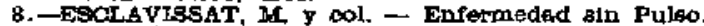
A proposito de dos casos en wujeres jovenes. Arch. Inst. Card. Mexion. $27: 045,1957$.
9.-FROVIG, LOKBN. - The Spndrome of Oblters. tion of Anterial Branches of the Aortic Arch Due to Arterltis, Acta Fisychiatitc et Nourol. Goandinaplan. 26:313. 1951 .

10. JPMENTEZ OASALO, M. $\mathrm{y}$ DOL - Un caso de enJermedar sin pulso. Rep Olí. Espan $43: 146$, 1056.

11.-KOSZEWGKI, B - Pulkeless Dlaease Due to Bra chlal Artertils. Circulation. 19:406. 1957.

12. MOAIA, B. $y$ col. - Bindirome del Arco Adrtico. Rev. Art. Chandol. 23:101, 1256

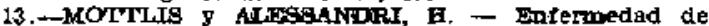
Taks yashu. Rev. Misc. Chlle. $86: 086$, 1058

14.-MOUQUIN. ERGVIGNES. - Un cas de syndrom de Taknyathu. Amflioration de la viston par 1 AOTH. Bull. et Mem. Boc. Med. Eop, Parls. 71: 1066, 1955 .

15.-MOURa et MIGOZZI. - La Maladie des Femmes Bans Pouls. Semain. Hop. Parts. 32:87, 1956.

16. - SEN GUPTA - Glosh Case of Reverse cositsction Syndrome. Brit, Eeant. J. 18:137, 1957.

17. उस्MMZU, K. - Puilseless Diserse (ebstracted). J. A. M. A. 115:1005, 1951 .

18.-WACIEMNIGR, F. J. - The Ocular Leslons of Pulseles Dhgogse. Am. J. M. Sc. 200idl, 1958.

19.-WARREN and TRTHDNAN, L. J.-Pulseless Disease and Carotid Artery Thrombosis Burglas Conkideratlons. The New ingland $J$, of Nied. $44: 645,1957$

20. -ROSS, Mc KUSICX, M, D. - Aortic Areh Sydaroms. Arch. Int Med. 92:701, 1053 